\title{
Animal models of vertical bone augmentation (Review)
}

\author{
ZEPENG ZHANG $^{1}$, YAXIN GAN ${ }^{1}$, YARONG GUO $^{1}$, XUGUANG LU $^{1}$ and XIANQI LI ${ }^{1,2}$ \\ ${ }^{1}$ Department of Oral and Maxillofacial Surgery, Shanxi Medical University School and Hospital of Stomatology, \\ Taiyuan, Shanxi 030001, P.R. China; ${ }^{2}$ Department of Oral and Maxillofacial Surgery, School of Dentistry, \\ Matsumoto Dental University, Shiojiri, Nagano 399-0781, Japan
}

Received February 8, 2021; Accepted June 10, 2021

DOI: $10.3892 / \mathrm{etm} .2021 .10351$

\begin{abstract}
Vertical bone augmentation is an important challenge in dental implantology. Existing vertical bone augmentation techniques, along with bone grafting materials, have achieved certain clinical progress but continue to have numerous limitations. In order to evaluate the possibility of using biomaterials to develop bone substitutes, medical devices and/or new bone grafting techniques for vertical bone augmentation, it is essential to establish clinically relevant animal models to investigate their biocompatibility, mechanical properties, applicability and safety. The present review discusses recent animal experiments related to vertical bone augmentation. In addition, surgical protocols for establishing relevant preclinical models with various animal species were reviewed. The present study aims to provide guidance for selecting experimental animal models of vertical bone augmentation.
\end{abstract}

\section{Contents}

1. Introduction

2. Different experimental models of vertical bone augmentation

3. Discussion

4. Conclusion

\section{Introduction}

In recent years, dental implants have become the preferred treatment for missing teeth. However, successful implantation relies on sufficient alveolar bone to provide primary stability

Correspondence to: Dr Xianqi Li, Department of Oral and Maxillofacial Surgery, School of Dentistry, Matsumoto Dental University, 1780 Gobara Hirooka, Shiojiri, Nagano 399-0781, Japan E-mail: xianqi.li@mdu.ac.jp

Key words: animal models, vertical bone augmentation, bone ring technique, guided bone regeneration, biomaterials, preclinical experiment in order to ensure the successful osseointegration of a dental implant (1). Alveolar bone resorption or defects are frequently caused by bone remodeling following tooth extraction, periodontitis, trauma and/or tumors (2); these changes cause loss of alveolar height and width. Commonly used techniques for the reconstruction of alveolar defects, including guided bone regeneration (GBR), bone splitting and free bone block transplantation, are able to effectively reconstruct the width of the alveolar bone; however, vertical bone augmentation remains a challenge in dental implantology (3).

Currently available vertical bone augmentation techniques include GBR, onlay bone grafting, distraction osteogenesis (DO) and the bone ring technique. Although these may be useful for certain patients, they have various disadvantages, such as unpredictable surgical effects and more frequent post-operative complications (4). GBR is the most commonly used vertical bone augmentation technique in which barrier membranes are used in areas of bone defects to isolate epithelial and connective tissue; the osteoblasts subsequently regenerate new bone without the intervention of other cells (5). GBR usually uses granular bone for bone regeneration. A notable disadvantage of particulate bone graft material is poor mechanical stability (6), which frequently requires an additional membrane or titanium mesh for binding; furthermore, with this technique, it is difficult to maintain the initial height (7). Bone blocks have a specific volume and resistance that may prevent the compression of internal soft tissue and maintain inner space for bone regeneration (8). Onlay bone grafting uses free bone blocks harvested from donor sites in the patient (e.g., mandible ramus, chin, calvarium, proximal tibia and iliac crest), which are fixed onto the recipient site with screws for vertical bone augmentation (9). However, this technique is associated with several limitations, such as a high absorption rate and postoperative pain, as well as nerve and soft tissue injuries (10). DO refers to the use of a distraction device to gradually separate partially or completely fractured alveolar bone and form new bone in the distraction gap (11). $\mathrm{DO}$ is able to achieve more vertical bone gain compared with GBR and onlay bone grafting but remains technically sensitive and is associated with a greater number of complications (12); furthermore, patients tend to find it difficult to tolerate this procedure. The bone ring technique has recently emerged and has achieved excellent outcomes for vertical bone augmentation $(13,14)$. This technique, which uses a ring-shaped bone grafting material, is a one-stage approach to vertical 
bone augmentation with simultaneous insertion of dental implants (15). This technique may reduce overall implant treatment time compared with other techniques $(16,17)$.

Autogenous grafts remain the 'gold standard' for vertical bone augmentation due to their osteogenic, osteoconductive and osteoinductive properties (18). Other bone substitute materials, such as allogeneic and xenogeneic grafts, synthetic bone substitutes and biodegradable metals (19), have also been introduced for bone repair; however, these materials are associated with several limitations (20). These aforementioned technologies are not able to completely solve the problem of vertical bone defects. Hence, it is necessary to develop novel techniques and biomaterials to achieve vertical bone regeneration. Suitable experimental/preclinical models are important for scientists to evaluate the bone regeneration and biomechanical properties of new biomaterials, as well as the application of related surgical techniques (21). The present review discusses vertical bone augmentation experiments performed in various species and different experimental sites and evaluates the advantages and disadvantages of each species with regard to vertical bone augmentation. The aim was to provide guidance for selecting suitable experimental animal models for vertical bone augmentation.

\section{Different experimental models of vertical bone augmentation}

A number of animal models, including rat, rabbit, dog, pig and sheep, have been developed to simulate the human in vivo environment to test novel biomaterials, compare different bone substitute biomaterials or develop novel osteogenic technologies. The selection of an appropriate animal model for vertical bone augmentation should be based on the following considerations: i) Appropriate simulation of human alveolar bone physiology and oral environment; ii) costs of acquisition and care; iii) stable and uniform genetic background of the experimental animals; iv) easy availability and comparison of experimental results; v) operability of the experimental animals; and vi) socio-ethical acceptability $(22,23)$. However, the selection of animal models for vertical bone augmentation should not only consider the characteristics of the bone graft material. It is also important to take into consideration the selection of the surgical method for vertical bone augmentation. The most frequently used animal models for vertical bone augmentation are discussed below.

Rat models. Rats are cost-effective, easy to handle, have a well-defined genetic background and may be genetically engineered to construct different pathological states that simulate different clinical conditions (24). However, rats have a higher metabolic rate compared with humans. The rate of new bone formation is influenced by the metabolic rate; thus, osteogenic potential is higher in rats compared with that in humans (25). The composition and bone mineral density of rat cortical bone differ greatly from that of humans and the levels of insulin-like growth factor-1 are significantly higher compared with those in human cortical bone (26). However, the operability and operating space of rats are limited by their small size. In addition, it is difficult to remove an equal amount of autologous bone as a control group in this species.
Application of rat models for vertical bone augmentation. Rat models have been used to test the osteoconduction of novel bone graft materials. The most common experimental site is the calvaria. For instance, Hoornaert et al (27) prepared circular grooves with trephine on the calvarial bone of rats. This was followed by a polymethylmethacrylate (PMMA) shell to construct the vertical GBR model. The authors then compared the vertical bone regeneration capacity of deproteinized bovine bone matrix (DBBM) and synthetic biomimetic calcium phosphate. Zhang et al (28) placed Teflon rings into the circular grooves made with a trephine on the calvaria of the rats. The authors then investigated the effects of doxycycline-doped Bio-Oss on vertical bone augmentation. In another study, Shino et al (29) filled a secluded space created by a plastic cap in the calvaria of rats with melatonin powder and successfully observed new bone formation in this animal model. By contrast, Zigdon et al (30) secured rigid gold domes on the rat calvaria by using fixation screws via anchoring rings to compare the osteogenic potential of Bio-Oss collagen, $\beta$-tricalcium phosphate $(\beta$-TCP) and a collagen sponge. Subsequently, Zigdon-Giladi et al $(10,31)$ successfully observed and compared the vertical osteogenic effects of $\beta$-TCP scaffolds with bone marrow-derived stem cells (BMSCs), osteogenic-transformed BMSCs and endothelial progenitor cells in the calvaria vertical GBR (VGBR) rat model; when compared to the scaffold alone, new bone height doubled following cell transplantation. In another study, Hao et al (32) developed an injectable alginate gel system, in which strontium hydroxyapatite (HA) was used to improve the osteogenic properties of the gel; the authors injected the gel into the rat subperiosteally using a 25-gauge needle. Eight weeks later, the vertical bone height was 2.3-fold greater than the calvarial thickness. Subsequently, Hao et al (33) introduced different doses of simvastatin into a strontium HA alginate gel that was then injected into the rat subperiosteally. The authors demonstrated that the application of low-dose simvastatin $(0.02 \mathrm{mg})$ in a strontium HA/alginate in situ gel system was able to promote the formation of new bone.

Protocol for developing a calvaria VGBR rat model. The calvaria VGBR model is based on the principle of GBR and has been successfully used to test biomaterials (Table I). From a developmental perspective, the calvaria and jaw are plate-like bones with embryonic homology (34). However, from a translational perspective, the calvaria is similar to an atrophic mandible (35); therefore, animal calvaria may be used as an experimental site to test bone materials for vertical bone augmentation $(36,37)$. The advantages of the calvaria model include adequate surgical access, a clear surgical field of view, ease of operation, uncomplicated postoperative care and good reproducibility (38). As only one side has osteogenic potential and bone marrow space is limited, the true osteoconductive properties of various materials may be readily determined (31). However, the calvaria VGBR model lacks the mechanical stress required for new bone maturation (39).

Adult healthy rats are normally selected as the experimental subjects. The forehead of the rat is first shaved and disinfected under total anesthesia. Depending on the size of the shell, a U-shaped incision (30) or a mid-sagittal linear incision (40) is made to lift a full-thickness flap and expose the calvarial bone. Small cortical bone perforations, which may 


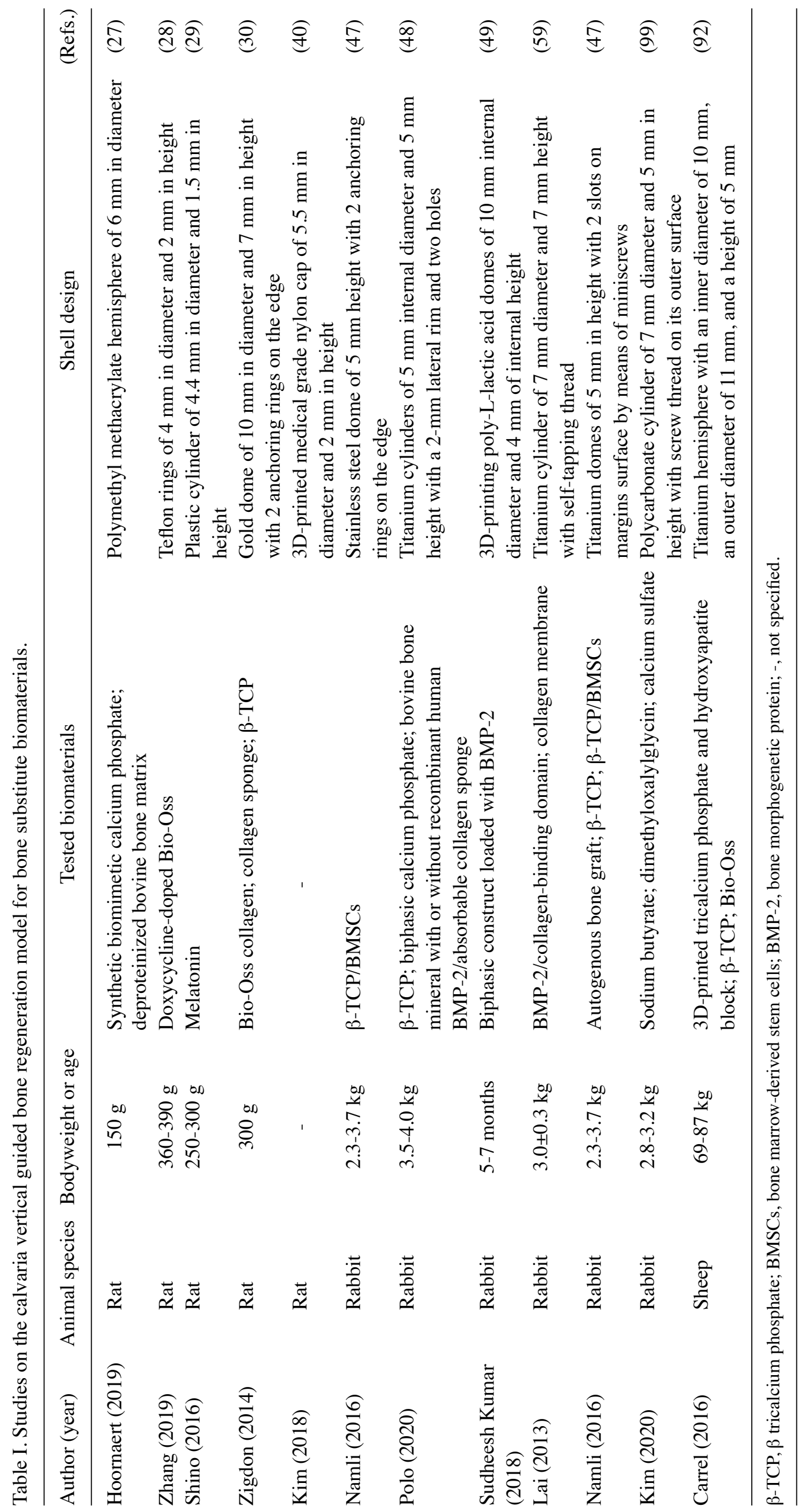


draw blood from the bone marrow into the space under the domes and accelerate bone formation $(41,42)$, are then drilled under cooling conditions with sterile saline and a round bur. The shell is then filled with biomaterials and fixed to the rat calvaria directly using screws via an anchor ring $(30,40,43)$ or placed on a circular groove (27-29) that is made on the rat calvaria using a trephine under profuse irrigation with sterile saline. As the rat calvaria is thin, care should be taken not to damage the endocranium. These shells simulate the barrier membrane used in clinical GBR practice and may provide a stable space for particulate biomaterials (27).

Rabbit models. Rabbits are the most commonly used laboratory animals, as they are easy to acquire, house and handle. Rabbits have a short developmental cycle, reaching skeletal maturity at 6 months of age (44). Furthermore, rabbits exhibit faster cortical bone remodeling and bone turnover compared with rodents or primates (45). In addition, rabbits have large amounts of adipose tissue in the medullary spaces of the mandible (46). These make it difficult to extrapolate experimental observations to human clinical conditions.

Application of the rabbit model for vertical bone augmentation. The common experimental site in the rabbit model is the calvaria. Several studies have used the rabbit calvaria VGBR model to test granular bone graft materials. For instance, Namli et al (47) evaluated the vertical bone regeneration capacity of $\beta$-TCP with BMSCs and compared this with autogenous bone graft in the rabbit calvaria VGBR model. In another study, Polo et al (48) compared the bone augmentation of three materials with recombinant human bone morphogenetic protein-2 (rhBMP-2)/absorbable collagen sponge using this model. The results demonstrated that biphasic calcium phosphate and $\beta$-TCP were suitable carriers for rhBMP-2/absorbable collagen sponge for vertical bone augmentation. In another study, Sudheesh Kumar et al (49) placed a 3D-printing biphasic construct loaded with BMP-2 in the same model. In vitro, the upregulation of bone markers was observed. However, the vertical bone formation was limited in vivo. The calvaria of rabbits may also be used to test onlay grafts. Lee et al (50) quantified the healing following vertical augmentation of allogenic bone blocks with/without rhBMP-2 on rabbit calvaria by radiographic and histological analyses. Sheikh et al (51) further compared the vertical bone height of two types of monetite (a bioresorbable dicalcium phosphate) block bones produced by wet or dry heating methods in the calvaria of rabbits. In another study, Sheikh et al (52) incorporated C3 (a bisphosphonate and a potent bone-activating EP4 receptor agonist) into brushite- and monetite-based block grafts and then implanted these in the rabbit calvaria. After 12 weeks, both grafts (loaded with C3) resulted in better new bone regeneration compared with grafts without C3. Furthermore, monetite-based blocks produced more vertical bone height compared with brushite. Tamimi et al (53) used positron emission tomography (PET)-computed tomography (CT) to investigate the metabolic activity of new bone in block bones and determined that calvarial bone metabolism exhibited higher levels of activity in the inferior and lateral areas of the block bones. The authors also reported that PET-CT analysis may be a promising tool for assessing bone viability in bone grafts.
Other studies have used the tibia and mandible as experimental sites. The tibial model may be utilized for experiments related to the bone ring technique and onlay grafting. Draenert et al (54) simultaneously implanted a biphasic implant (consisting of ring-shape cancellous bone-derived material and a commercial dental implant) into the rabbit tibia with vertical bone augmentation to evaluate bone healing and the proof of principle for the bone ring technique. Histological examination revealed that all specimens exhibited new bone formation in the scaffold, as well as good osseointegration of the dental implant. Kim et al (55) successfully assessed vertical bone augmentation using block form DBBM, with or without rhBMP-2, in rabbit tibia by means of radiographic, histological and immunohistochemical analyses. The results demonstrated that DBBM covered by absorbable collagen sponge embedded with rhBMP-2 was able to accelerate new bone formation. Furthermore, higher expression levels of vascular endothelial growth factor (VEGF) were detected in this group compared with other groups. In another study, Veis et al (56) placed block form DBBM using orthopedic mini-plates and particulate DBBM using custom-made perforated metallic cubes into the lateral mandible of rabbits. Histomorphometry demonstrated that vertical bone augmentation outcomes were considerable in the two types of DBBM. Pripatnanont et al (57) performed periosteal distraction osteogenesis using a modified hyrax device (a type of orthodontic device for children) in rabbit mandibles. The modified hyrax successfully promoted new bone formation, although it was difficult to control the direction of new bone formation, as most of the new bone was growing laterally to the mandible rather than vertically.

Protocol for developing rabbit calvaria and tibia models. The VGBR model of rabbit calvaria is similar to that developed for rats. Marger et al (35) described the detailed protocol for using rabbit calvaria as a VGBR model. The surface area of the rabbit calvaria is larger compared with that of the rat and up to four experimental groups may be compared in the same animal $(58,59)$. Compared with the rat model, the rabbit model is also advantageous because it is possible to take an equal amount of autologous bone as a control group (47). The rabbit calvaria onlay model for block bone material is easier to apply compared with the VGBR model; block bone material is placed on the calvarial bone and fixed with screws.

The rabbit tibia model provides the opportunity of analyzing bone regeneration in a relatively confined environment using larger onlay bone graft materials; it is also possible to use conventional implant sizes using the bone ring technique (60). Compared with the calvaria, the tibia has a geometric curvature that is more similar to that of the mandible (61). The protocol for establishing a tibia model is based on methodology that was previously reported by Kim et al (55). Following anesthesia, an incision $(4 \mathrm{~cm}$ in length) is made on the proximal tibia running up to the tibial diaphysis; then, a full-thickness flap is lifted, thus revealing the lateral tibial bone. The receptor bed may then be perforated with round burs under cooling conditions with sterile saline. Tow grafts are then fixed with bi-cortical screws that pass through the cortical bone and into the marrow cavity in each tibia. Finally, absorbable sutures are used to close the soft tissues and nylon threads are used to suture the skin. 
Canine models. Canine models are widely used in dental research. Dogs and humans have several similarities with regard to bone, including similar secondary osteons and comparable intracortical remodeling (62). Canines and humans also have similar levels of gray matter, collagen and insulin-like growth factor-1 in cortical and cancellous bone (26). Furthermore, it has been determined that the bone mineral density of the mandible and periodontal tissue of beagle dogs is similar to that of humans (63). However, annual whole-body trabecular bone turnover rates are higher in dogs (64). Furthermore, the rate of trabecular bone turnover in dogs is highly variable, both within and between individual animals (64).

Application of canine models for vertical bone augmentation. The common experimental site used in canine models is the mandible. This strategy may be used in preclinical studies to determine the efficacy and applicability of biomaterials, surgical methods and medical devices with different vertical bone augmentation techniques. For instance, Carrel et al (65) extracted the bilateral premolars and first molars of a dog's mandible under general anesthesia; three months later, the authors then performed onlay bone grafting covered with a collagen membrane using 3D-printing calcium phosphate porous blocks in the edentulous area. Teng et al (66) coated a layer of calcium phosphate with BMP-2 on DBBM blocks. The blocks were fixed onto the atrophic alveolar ridge of beagles. Those studies demonstrated that calcium phosphate promoted the osteoconduction of BMP-2 to the upper part of the bone block. Several studies have investigated autogenous tooth roots (with or without autoclavation) (67) as block grafts for vertical bone augmentation by means of micro-CT (68) and histomorphometrical (69) analyses in the mandible of beagles. Both autogenous tooth roots were gradually replaced by newly formed bone. Of note, more vertical bone gain was observed in the autoclavation group (69); however, the untreated group exhibited better osseointegration of the implants (67). Hsu et al (70) used titanium mesh and human allografts in combination, with or without rhBMP-2, as the GBR in the mandibles of beagle dogs and then compared hard tissue outcomes between the two groups. In another study, Xuan et al (71) successfully compared new bone generation in Bio-Oss blocks between flap and tunnel procedures using the onlay grafting technique in the canine mandible. The results of that study demonstrated that new bone generation using tunnel procedures was superior to that using flap procedures. In other studies, Nakahara et al $(16,17)$ determined the effect of implant placement using the bone ring technique in the edentulous region of the dog mandible. Histomorphometric and micro-CT analyses revealed that the total area of mineralized bone was similar between the two groups; the single-stage procedure achieved equally efficient osseointegration compared with the two-stage procedure. Furthermore, there was a higher extent of residual bone graft in the single-stage group but a higher extent of new bone formation in the two-stage group. Esposito et al (72) also performed DO using a modified intraosseous distractor for vertical bone augmentation in the posterior mandible of dogs. In another study, Terbish et al (73) evaluated the effect of rhBMP-2 injected after a DO procedure in beagle dogs. Injection of rhBMP-2 significantly increased the bone volume and improved both the width and height of the alveolar ridge. Kaner et al $(74,75)$ performed soft tissue expansion (STE) prior to vertical bone augmentation to evaluate whether this technique may improve the outcomes of vertical bone regeneration in the canine mandible. In the test group, self-filling osmotic tissue expanders were placed in the mandible of the dogs. After 5 weeks, the test group underwent vertical bone augmentation with only one horizontal incision made above the alveolar ridge; in the control group, an additional vertical release incision was made. There was no wound dehiscence at the STE sites; however, 8 cases of wound dehiscence were observed at the control sites. Microcirculation measurements revealed significantly better perfusion in the experimental group compared with that in the control group (75). The expanded soft tissue appeared to reduce pressure on the scaffold and the area of bone was larger in the experimental group than in the control group.

Several studies refer to other experimental sites. Sawada et al (76) inserted three types of biological bone block (DBBM, $\beta$-TCP and $\alpha$-TCP/HA) into the calvarial bone defects in beagle dogs. During the healing period, all bone blocks retained their original form, although the amount of new bone formation in the substitute was limited. Bianchini et al (77) inserted bovine bone screws to guide vertical bone regeneration in the tibia of beagle dogs. The results demonstrated that bovine bone screws with titanium-reinforced membranes were able to provide an environment for new bone formation and significantly enhance vertical bone gain.

Protocol for developing a canine mandibular alveolar bone atrophy model. Beagles are known to have good genetic stability, good reproducibility in different individuals in the same experimental condition, and they are easy to tame and adapt to different environments. They also have periodontal tissues that are similar to those of humans. The beagle mandible has four premolar teeth as well as three molars and may therefore provide a wide range of vertical bone defects by extracting premolar or molar teeth. The highly tractable nature of dogs is also beneficial to postoperative healing (78). Based on these points, the beagle mandible model is the best model for vertical bone augmentation and is widely used in experimental studies of vertical bone augmentation. This model may be used in preclinical studies to determine the efficacy and applicability of vertical bone augmentation techniques, surgical procedures and medical devices. However, due to social-ethical concerns, it is becoming increasingly difficult to obtain ethical approval for experiments using dog models.

The protocol used to create a canine mandible model, as reported by Parvini et al (69), is reviewed below. Adult healthy beagles are used as the experimental subjects. In the first round of surgery, the dogs are placed under total anesthesia; a full-thickness flap of the bilateral mandible is then incised and elevated. The premolars and molars are extracted; subsequently, the box-type vertical defect is prepared using a straight fissure carbide bur under cooling conditions with sterile saline. Final sutures are used to close the wound and the dogs are allowed to heal for 12 weeks. In the second round of surgery, bilateral vestibular incisions are created and the flaps are retracted to expose the vertical defect for vertical bone augmentation surgery. Vertical alveolar bone defects may be designed according to different sizes of bone 
grafting materials or vertical bone augmentation techniques. Teng et al (66) extracted bilateral dog mandibular premolars; loss of the vertical alveolar bone was observed after 1 month of healing. In another study, Khojasteh et al (79) extracted the bilateral first and second mandibular premolar teeth bilaterally in dogs; after 4 weeks of soft tissue healing, critical size defects were evident. The authors were then able to perform vertical bone augmentation. The different sizes of vertical defects in the canine mandibular model are summarized in Table II. This animal model may be divided into two phases: Defect creation and vertical bone augmentation. Therefore, the application of this animal model requires a long experimental duration.

Porcine models. Porcine bone exhibits similarities to human bone in terms of both bone mineral density and bone mineral concentration (26). Pigs have a comparable bone remodeling process and lamellar bone structure to that of humans $(80,81)$ and mature animals have a well-developed Haversian system (82). Of note, the bone regeneration rate of pigs is similar to that of humans (1.2-1.5 vs. $1.0-1.5 \mathrm{~mm} / \mathrm{day}$, respectively) (83). However, the trabecular network of pigs is denser (80). Pigs are also more difficult to handle, noisy and aggressive (84); furthermore, oral hygiene cannot be ensured (85).

Application of porcine models for vertical bone augmentation. Schorn et al (85) applied disc-shaped collagenous scaffolds with/without rhBMP-2 and/or VEGF around the implants; then, the implants were combined with a scaffold and inserted into the mandible and tibia of mini pigs. The results demonstrated that insoluble disc-shaped collagenous bone matrix was able to maintain acceptable volume stability with low resorption after 12 weeks and that the collagenous scaffolds containing rhBMP-2 and VEGF formed more new vertical bone around the implants. Implants inserted into the tibia had higher rate of success compared with implants inserted into the lower jaw. Moest et al (86) used pig skull models to compare consolidation between allogeneic and autologous bone blocks for vertical bone augmentation. The quantity and quality of the two different blocks were investigated by histological analysis, histomorphometry and immunohistochemistry. In that study, less new bone and lower expression levels of collagen type I and osteocalcin were observed in allogeneic bone grafts compared with those in autologous bone grafts. In another study, Freilich et al (87) developed a novel custom scaffold retainer (umbrella) that aimed to guide new bone with minimal technique sensitivity (simplified operation and reduced postoperative complications) and successfully grow a new layer of bone in the mandibles of mini pigs. In another study, Wen et al (88) evaluated the efficacy of various scaffold systems and an 'umbrella' with or without non-glycosylated rhBMP-2 that was used to increase vertical alveolar bone in the mini pig model. Clinical observations further revealed that soft tissue healing was significantly better in groups with BMP-2 than in those without BMP-2.

Protocol for developing a porcine mandible and tibia model. Partly due to the fact that the use of the canine model has been limited by socio-ethical issues and also due to the observation that porcine bone is highly similar to human bone, pigs are gradually becoming a bridge for the translation of research results from small animal models to human clinical trials. Domestic pigs are not favored by researchers due to their rapid growth rate and large size, and mini pigs are considered to be more suitable as experimental animals.

A commonly used protocol for developing a mandible and tibial model in pigs was reported by Schorn et al (85). In brief, all animals subjected to surgery received general anesthesia. The mandibles were cleaned with antiseptic mouthwash and the two premolar teeth and the first molar teeth were removed. Mini pigs are known to produce excessive saliva; thus, saliva-proof wound closure was performed by interrupted sutures. To aid soft tissue healing 3 months after tooth extraction, dental implants with disc-shaped collagenous scaffolds were inserted into the mandible and the tibia. After 2, 4 and 12 weeks, the animals were sacrificed, and tibial and mandibular block specimens were harvested for histological analysis and histomorphometry.

Sheep models. The turnover and bone modeling rate in sheep are similar to those of humans (89). These facts make sheep a promising animal model for bone regeneration research. The main advantage of sheep is that their bones are sufficiently large to allow multiple conditions to be compared simultaneously (90). Compared with human bones, the bones of sheep have a similar macrostructure but a different microstructure. Sheep bones consist predominantly of primary bone structure; by contrast, human bones consist mainly of a secondary bone structure (91). In addition, sheep undergo seasonal periods of bone loss; therefore, control animals may spontaneously lose and regain bone during winter (82).

Application of the sheep model for vertical bone augmentation. The sheep calvaria VGBR model was previously used by Carrel et al (92) to perform a histomorphometric analysis of a 3D-printed TCP/HA porous block. They placed three different bone substitutes (3D-printed block, particulate Bio-Oss or $\beta$-TCP) in titanium shells; these were then immediately placed on the sheep calvaria. After 8 weeks, new bone was observed at the top of the 3D-printing block but at the bottom of all other materials. After 16 weeks, the spaces of all three materials were almost entirely filled with new bone. In another study, Jinno et al (89) fitted implants with three materials in the form of ring-shaped bone blocks (autogenous bone, Bio-Oss collagen or a resorbable biphasic ceramic) into the lateral margins of the body of the sheep mandible and subsequently evaluated the changes in volume and osseointegration in the bone rings by $3 \mathrm{D}$ imaging and histomorphometric analysis. All ring-shaped blocks were well-fixed and stable within the implants, although the volume of the grafts was reduced in all of the groups. Compared with the other materials, dense cortical bone blocks yielded the best results in terms of volume maintenance. In another study, Benlidayi et al (93) successfully used the sheep model to compare the effects of autogenous and allograft bone rings in iliac vertical bone defects. Histological analysis revealed that allogenic bone rings were completely replaced with new bone after 8 months. Therefore, bone ring technology constitutes a vertical bone augmentation procedure with reliable and predictable outcomes. 


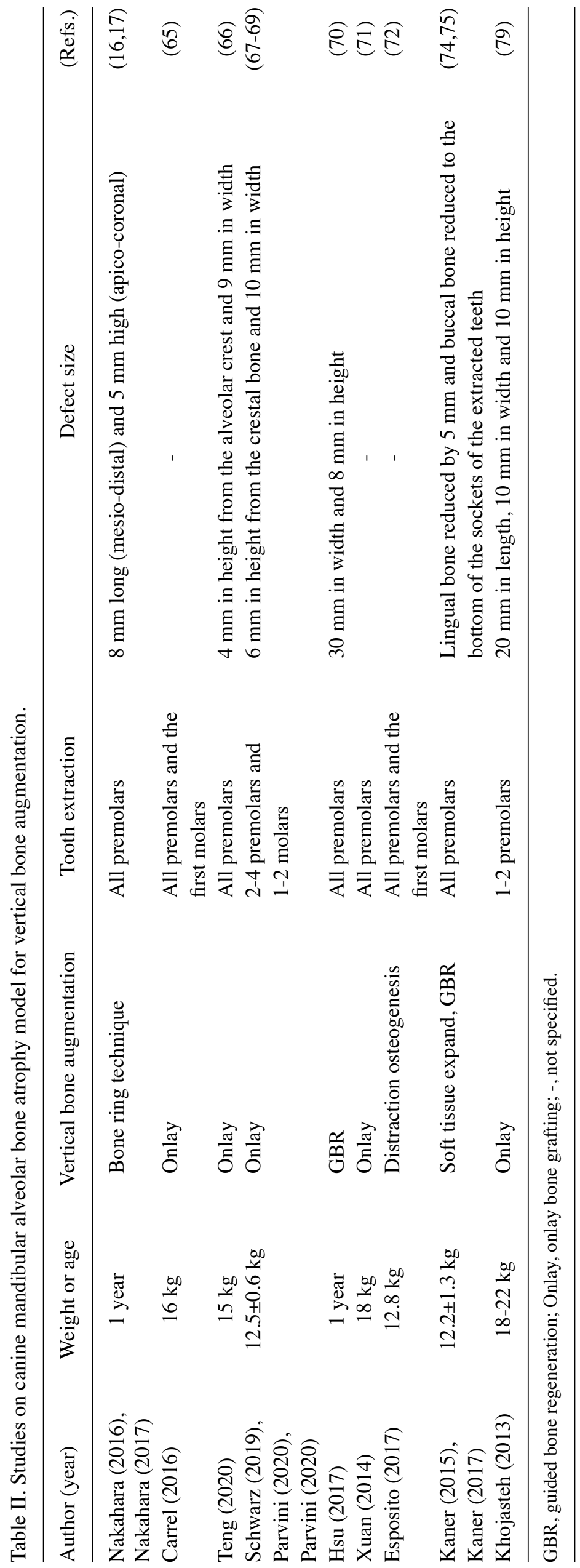


Protocol for developing a sheep iliac model. The sheep calvarial model may be used to test bone biomaterials with the same level of efficiency as that of the rat or rabbit calvaria VGBR model $(90,92)$. However, the masticatory activity of sheep may result in flap dehiscence, bone graft failure or implant failure following vertical bone augmentation (93). Therefore, the alveolar ridge cannot be used as the experimental site and only the lateral edge of the mandible should be used; this issue imposes limitations in terms of the type of operation used and the size of the bone graft materials.

The sheep iliac crest provides a large space for manipulation, thus allowing multiple experimental sites to be set up in a single animal. In addition, the iliac crest represents an ideal site for bone grafting and implantation in sheep (93). The protocol for establishing an iliac model was described by Colpak et al (94). The surgical area is first shaved and sterilized under general anesthesia. A skin marker is then used to mark the surface location of the iliac crest body. Next, a $10-\mathrm{cm}$ horizontal skin incision is made in the anterior third, followed by blunt dissection of the subcutaneous fascia and tissues. An oblique incision is then made on the muscle attached to this region. Finally, the iliac crest is exposed using a periosteal elevator for vertical bone augmentation.

\section{Discussion}

Several previous studies have summarized the animal models used for studying the repair of bone (62) and cartilage defects (95) and recommended histological analysis of animal experiments (96). In the present study, recent articles on vertical bone augmentation experiments performed in animal models using rats, rabbits, dogs, pigs and sheep were summarized and discussed. References and suggestions for the selection of animal models for vertical bone augmentation according to different experimental purposes are listed in Table III. The review by Wancket (62) discussed similarities and differences between the composition and remodeling of bones of different species and human bones. However, the present review mainly lists the application scenarios in different animals and describes the construction process of different animal models in detail. At the same time, the animal model of vertical bone augmentation is different from that of bone defect and nonunion. For instance, the small animal critical size defect model mentioned in the article by Wancket (62) is not suitable for the study of vertical bone augmentation, while the calvaria VGBR model discussed in the present review is more suitable for the study of bone graft materials for vertical bone augmentation.

The calvaria is a common experimental site to test and compare the osteogenic ability of different bone graft materials for vertical bone augmentation, including the calvaria VGBR and calvaria onlay models. The reasons for and advantages of using the calvaria as the experimental site have been mentioned above. In the VGBR model of rats (27), rabbits (97) and sheep (90), the granular DBBM displayed a similar histological structure. The bone augmentation area is composed of mature new bone close to the calvaria, with residual DBBM granules and vascularized connective tissue containing mono- and multinuclear cells. However, small animals (rats and rabbits) exhibited a faster rate of new bone formation compared with sheep. Histological observation of the blank group revealed that the osteogenic potential of the rabbit skull was the highest and moderate new bone formation was observed; a small amount of new bone was observed in rats, whereas in sheep, new bone formation was almost absent. Cortical bone perforation in GBR has always been a controversial issue (98). Certain studies have indicated that cortical bone perforation may accelerate the formation of new bone and exerts no harmful effect on the experimental results, although it cannot significantly increase the total amount of new bone $(41,98)$. It is suggested that cortical bone perforation should be performed in VGBR models, but the location and number of perforations should be consistent to ensure the objectivity of the experiment. The barrier shell design of the VGBR model is described in detail in Table I. Titanium shells have good biocompatibility and new bone is able to grow along the titanium shell and be closely associated with bone tissue. However, it is difficult to separate the sample from the titanium shell; this limits the detection methods used for these samples (59). Polo et al (97) designed a removable titanium dome; they then performed micro-CT analysis. Non-metallic barrier shells, including PMMA (27), Teflon (28), plastic (29), polyether ether ketone (35) and polycarbonate (99) shells, are biocompatible and do not cause any damage to the experimental animals or adversely affect the results. Histological observations indicated that the new bone does not touch the shell, thereby avoiding problems related to the separation of the samples from the shells. In addition, 3D printing technology has been successfully applied to the design of the VGBR animal model $(40,49,99)$, which may considerably reduce the time and cost compared with traditional casting metal barrier shells. The calvaria onlay model is commonly used to compare and detect different block bone grafts. Block bone has a specific volume and resistance, which may prevent internal soft tissue from being compressed and is able to maintain internal space for bone regeneration. The majority of block bone grafts are able to maintain the original shape and fuse with the surface of the original bone. The new bone is mainly formed adjacent to the fixation screw and along the lower border of the bone block $(50,53,100)$. Adipose tissue was observed in certain experiments $(50,100)$, but not in the VGBR model.

The mandibular alveolar bone atrophy model in beagle dogs is widely considered to represent the best model for simulating human vertical bone augmentation surgery. As this model is able to closely simulate mandibular defects in human patients, it is suitable for determining and comparing osteogenesis in different types of vertical bone augmentation surgery and related surgical procedures, medical devices and bone graft materials. Wound dehiscence is the most common complication in this model; this complication may lead to wound infection and exposure of the bone graft material. Soft tissue follows the contour of the underlying bone; severe resorption of the jaw may lead to a reduction in soft tissue, thus affecting the tension-free closure of bone augmentation surgery (101). Kaner et al (75) performed STE prior to vertical augmentation, which may reduce the risk of wound dehiscence and significantly increase the new bone area. The difference between the mandibular alveolar bone atrophy model and the calvaria VGBR model is that the implant 


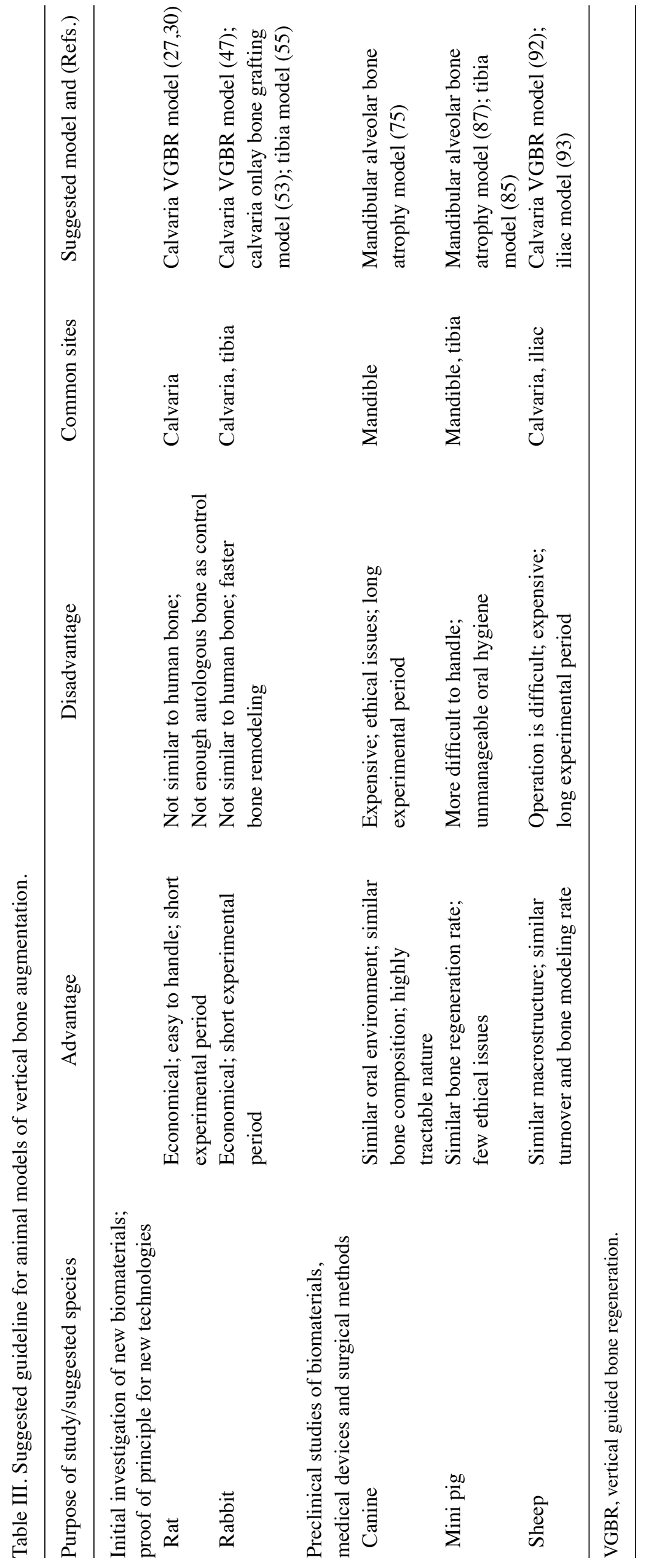


is in direct contact with the soft tissue. Carrel et al (65) performed onlay grafting using 3D-printed TCP/HA and histologically observed soft tissue ingrowth that degraded the edge of the bone block. Compared with the calvaria model, the bone healing of the canine mandible model more closely resembles that of humans and the Haversian system may be observed (70). However, the use of the canine model is limited by socio-ethical issues and the mini pig model appears to be the most promising alternative. The mandible defect model requires tooth extraction surgery with trimming of the vertical defect. Vertical bone augmentation may only be performed after a period of soft tissue healing. However, there are two disadvantages to this model: The long experimental duration and the high cost.

The tibial model in rabbits and pigs may be selected for studies using the bone ring technique (85) and onlay grafting (55). The tibial model provides the opportunity for bone regeneration analysis in a relatively confined environment using larger onlay bone graft materials and may accept conventional implant sizes for the bone ring technique. The iliac bone in sheep provides a large surface area for experimental sites and may be used to compare multiple bone graft materials in a single subject $(93,94)$. However, this model is not commonly used; this is because it is associated with high cost and because it is more difficult to perform surgery to expose the iliac crest in this model.

The calvaria VGBR model is a suitable model to study biomaterials for vertical bone augmentation, which is relatively well-established for construction. However, the production of traditional casting metal-based barrier devices increases the cost. The successful application of 3D printing technology offers a more convenient and cheaper option. The mandibular alveolar bone atrophy model in beagle dogs is the best model for vertical bone augmentation. However, there are two issues with this model. First, wound dehiscence is the same as in the clinical situation, which will adverse impact the experimental results due to wound infection and bone graft material exposure. An effective method of soft tissue area preservation should be established in the beagle mandibular alveolar bone atrophy model. Furthermore, ethical issues limit its usage. Miniature pigs (e.g., Gottingen mini-pig), which were bred by miniaturized and standardized using domestic pigs and have higher social acceptability, are a promising model to replace dogs to construct mandibular alveolar bone atrophy model. However, it is difficult to manage the oral health of miniature pigs. In the future, it is esteemed to optimize the protocols for developing miniature pig models and solve the problems pertaining to oral hygiene. In recent years, several studies have also applied the sheep model to the study of vertical bone augmentation $(89,92,93)$. However, due to the chewing behavior of sheep, it is difficult to perform experiments on the alveolar ridge.

\section{Conclusion}

The optimal animal model for vertical bone augmentation should be selected according to the purpose of the experiment and the bone augmentation technique applied. Small animals, such as rats and rabbits, are cost-effective and easy to control; thus, the VGBR and onlay models based on the calvaria of small animals may be used during the initial investigation of a new material. The mandible model in beagle dogs is currently the best animal model for vertical bone augmentation; however, the use of the canine model is limited by socio-ethical factors. Miniature pigs represent a promising alternative to dogs for such studies.

It is also vital to consider that bone metabolism in animals differs from that in humans. Therefore, the results arising from animal experiments should be interpreted with caution and should not be directly extrapolated to human subjects.

\section{Acknowledgements}

Not applicable.

\section{Funding}

The present study was supported by the Scientific Research Project Plan of the Health Commission of Shanxi Province (grant no. 2017119).

\section{Availability of data and materials}

Not applicable.

\section{Authors' contributions}

Conception and design of the study: XLu. Literature research and analysis: ZZ and YGa. Drafting and writing of the manuscript: ZZ, YGa and YGu. Revision and editing of the manuscript: XLu and XLi. All authors read and approved the final manuscript. Data authentication is not applicable.

\section{Ethics approval and consent to participate}

Not applicable.

\section{Patient consent for publication}

Not applicable.

\section{Competing interests}

The authors declare that they have no competing interests.

\section{References}

1. Yim HJ, Lim HC, Hong JY, Shin SI, Chung JH, Herr Y and Shin SY: Primary stability of implants with peri-implant bone defects of various widths: An in vitro investigation. J Periodontal Implant Sci 49: 39-46, 2019.

2. Moy PK, Medina D, Shetty V and Aghaloo TL: Dental implant failure rates and associated risk factors. Int J Oral Maxillofac Implants 20: 569-577, 2005.

3. Urban IA, Montero E, Monje A and Sanz-Sánchez I: Effectiveness of vertical ridge augmentation interventions: A systematic review and meta-analysis. J Clin Periodontol 46 (Suppl 21): S319-S339, 2019.

4. Elnayef B, Monje A, Gargallo-Albiol J, Galindo-Moreno P, Wang HL and Hernández-Alfaro F: Vertical ridge augmentation in the atrophic mandible: A Systematic review and meta-analysis. Int J Oral Maxillofac Implants 32: 291-312, 2017.

5. Wang HL and Boyapati L: 'PASS' principles for predictable bone regeneration. Implant Dent 15: 8-17, 2006. 
6. Artzi Z, Nemcovsky CE, Tal H, Weinberg E, Weinreb M, Prasad H, Rohrer MD and Kozlovsky A: Simultaneous versus two-stage implant placement and guided bone regeneration in the canine: Histomorphometry at 8 and 16 months. J Clin Periodontol 37: 1029-1038, 2010

7. Kon K, Shiota M, Ozeki M and Kasugai S: The effect of graft bone particle size on bone augmentation in a rabbit cranial vertical augmentation model: A microcomputed tomography study. Int J Oral Maxillofac Implants 29: 402-406, 2014.

8. Kim JW, Jeong IH, Lee KI, Jung UW, Kim CS, Choi SH, Cho KS and Yun JH: Volumetric bone regenerative efficacy of biphasic calcium phosphate-collagen composite block loaded with rhBMP-2 in vertical bone augmentation model of a rabbit calvarium. J Biomed Mater Res A 100: 3304-3313, 2012.

9. Nkenke E and Neukam FW: Autogenous bone harvesting and grafting in advanced jaw resorption: Morbidity, resorption and implant survival. Eur J Oral Implantol 7 (Suppl 2): S203-S217, 2014.

10. Zigdon-Giladi H, Bick T, Morgan EF, Lewinson D and Machtei EE: Peripheral blood-derived endothelial progenitor cells enhance vertical bone formation. Clin Implant Dent Relat Res 17: 83-92, 2015.

11. Rachmiel A, Shilo D, Aizenbud D and Emodi O: Vertical alveolar distraction osteogenesis of the atrophic posterior mandible before dental implant insertion. J Oral Maxillofac Surg 75: 1164-1175, 2017.

12. Tamimi F, Torres J, Gbureck U, Lopez-Cabarcos E, Bassett DC, Alkhraisat $\mathrm{MH}$ and Barralet JE: Craniofacial vertical bone augmentation: A comparison between 3D printed monolithic monetite blocks and autologous onlay grafts in the rabbit. Biomaterials 30: 6318-6326, 2009.

13. Giesenhagen B, Martin N, Donkiewicz P, Perić Kačarević Ž, Smeets R, Jung O, Schnettler R and Barbeck M: Vertical bone augmentation in a single-tooth gap with an allogenic bone ring: Clinical considerations. J Esthet Restor Dent 30: 480-483, 2018.

14. Giesenhagen B, Martin N, Jung O and Barbeck M: Bone augmentation and simultaneous implant placement with allogenic bone rings and analysis of its purification success. Materials (Basel) 12: 1291, 2019.

15. Stevens MR, Emam HA, Alaily ME and Sharawy M: Implant bone rings. One-stage three-dimensional bone transplant technique: A case report. J Oral Implantol 36: 69-74, 2010.

16. Nakahara K, Haga-Tsujimura M, Sawada K, Kobayashi E, Mottini M, Schaller B and Saulacic N: Single-staged vs. two-staged implant placement using bone ring technique in vertically deficient alveolar ridges-Part 1: Histomorphometric and micro-CT analysis. Clin Oral Implants Res 27: 1384-1391, 2016.

17. Nakahara K, Haga-Tsujimura M, Sawada K, Kobayashi E, Schaller B and Saulacic N: Single-staged vs. two-staged implant placement in vertically deficient alveolar ridges using bone ring technique-Part 2: Implant osseointegration. Clin Oral Implants Res 28: e31-e38, 2017.

18. Sakkas A, Wilde F, Heufelder M, Winter K and Schramm A: Autogenous bone grafts in oral implantology-is it still a 'gold standard'? A consecutive review of 279 patients with 456 clinical procedures. Int J Implant Dent 3: 23, 2017.

19. Yang Y, Cheng Y, Peng S, Xu L, He C, Qi F, Zhao M and Shuai C: Microstructure evolution and texture tailoring of reduced graphene oxide reinforced $\mathrm{Zn}$ scaffold. Bioact Mater 6 1230-1241, 2021

20. Zeeshan S, Corneliu S and Michael G: Bone replacement materials and techniques used for achieving vertical alveolar bone augmentation. Materials 8: 2953-2993, 2015.

21. Donos N, Dereka X and Mardas N: Experimental models for guided bone regeneration in healthy and medically compromised conditions. Periodontol 68: 99-121, 2015.

22. Li Y, Chen SK, Li L, Qin L, Wang XL and Lai YX: Bone defect animal models for testing efficacy of bone substitute biomaterials. J Orthop Translat 3: 95-104, 2015

23. Newman E, Turner AS and Wark JD: The potential of sheep for the study of osteopenia: Current status and comparison with other animal models. Bone 16 (Suppl 4): 277S-284S, 1995.

24. Gomes PS and Fernandes MH: Rodent models in bone-related research: The relevance of calvarial defects in the assessment of bone regeneration strategies. Lab Anim 45: 14-24, 2011.

25. Schlegel KA, Lang FJ, Donath K, Kulow JT and Wiltfang J: The monocortical critical size bone defect as an alternative experimental model in testing bone substitute materials. Oral Surg Oral Med Oral Pathol Oral Radiol Endod 102: 7-13, 2006.
26. Aerssens J, Boonen S, Lowet G and Dequeker J: Interspecies differences in bone composition, density, and quality: Potential implications for in vivo bone research. Endocrinology 139: 663-670, 1998

27. Hoornaert A, Maazouz Y, Pastorino D, Aparicio C, de Pinieux G, Fellah BH, Ginebra MP and Layrolle P: Vertical bone regeneration with synthetic biomimetic calcium phosphate onto the calvaria of rats. Tissue Eng Part C Methods 25: 1-11, 2019.

28. Zhang P, Ding L and Kasugai S: Effect of doxycycline doped bone substitute on vertical bone augmentation on rat calvaria. Dent Mater J 38: 211-217, 2019.

29. Shino H, Hasuike A, Arai $Y$, Honda M, Isokawa $K$ and Sato $S$ : Melatonin enhances vertical bone augmentation in rat calvaria secluded spaces. Med Oral Patol Oral Cir Bucal 21: e122-e126, 2016.

30. Zigdon H, Lewinson D, Bick T and Machtei EE: Vertical bone augmentation using different osteoconductive scaffolds combined with barrier domes in the rat calvarium. Clin Implant Dent Relat Res 16: 138-144, 2014.

31. Zigdon-Giladi H, Lewinson D, Bick $T$ and Machtei EE: Mesenchymal stem cells combined with barrier domes enhance vertical bone formation. J Clin Periodontol 40: 196-202, 2013.

32. Hao J, Chou J, Kuroda S, Otsuka M, Kasugai S and Lang NP: Strontium hydroxyapatite in situ gel-forming system-a new approach for minimally invasive bone augmentation. Clin Oral Implants Res 26: 581-585, 2015.

33. Hao J, Chou J, Kuroda S, Otsuka M, Kasugai S and Lang NP: Injectable simvastatin gel for minimally invasive periosteal distraction: In vitro and in vivo studies in rat. Clin Oral Implants Res 29: 227-234, 2018.

34. Berendsen AD and Olsen BR: Bone development. Bone 80 : $14-18,2015$

35. Marger L, Barone A, Martinelli-Kläy CP, Schaub L, Strasding M, Mekki M, Sailer I, Scherrer SS and Durual S: Calvarial model of bone augmentation in rabbit for assessment of bone growth and neovascularization in bone substitution materials. J Vis Exp: Aug 13, 2019 doi: 10.3791/59976.

36. Verna C, Dalstra M, Wikesjö UM and Trombelli L: Healing patterns in calvarial bone defects following guided bone regeneration in rats. A micro-CT scan analysis. J Clin Periodontol 29: 865-870, 2002.

37. Lundgren D, Lundgren AK, Sennerby L and Nyman S: Augmentation of intramembraneous bone beyond the skeletal envelope using an occlusive titanium barrier. An experimental study in the rabbit. Clin Oral Implants Res 6: 67-72, 1995.

38. Bigham-Sadegh A and Oryan A: Selection of animal models for pre-clinical strategies in evaluating the fracture healing, bone graft substitutes and bone tissue regeneration and engineering. Connect Tissue Res 56: 175-194, 2015.

39. Zakaria O, Madi M and Kasugai S: A novel osteogenesis technique: The expansible guided bone regeneration. J Tissue Eng 3: 2041731412441194, 2012.

40. Kim JM, Kim JH, Lee BH and Choi SH: Vertical bone augmentation using three-dimensionally printed cap in the rat calvarial partial defect. In Vivo 32: 1111-1117, 2018.

41. Lee SH, Lim P and Yoon HJ: The influence of cortical perforation on guided bone regeneration using synthetic bone substitutes: A study of rabbit cranial defects. Int J Oral Maxillofac Implants 29: 464-471, 2014.

42. Majzoub Z, Berengo M, Giardino R, Aldini NN and Cordioli G: Role of intramarrow penetration in osseous repair: A pilot study in the rabbit calvaria. J Periodontol 70: 1501-1510, 1999.

43. Kinard LA, Dahlin RL, Henslee AM, Spicer PP, Chu CY, Tabata Y, van den Beucken JJ, Jansen JA, Young S, Wong ME et al: Tissue response to composite hydrogels for vertical bone augmentation in the rat. J Biomed Mater Res A 102: 2079-2088, 2014.

44. Gilsanz V, Roe TF, Gibbens DT, Schulz EE, Carlson ME, Gonzalez O and Boechat MI: Effect of sex steroids on peak bone density of growing rabbits. Am J Physiol 255: e416-e421, 1988.

45. Castañeda S, Largo R, Calvo E, Rodríguez-Salvanés F, Marcos ME, Díaz-Curiel M and Herrero-Beaumont G: Bone mineral measurements of subchondral and trabecular bone in healthy and osteoporotic rabbits. Skeletal Radiol 35: 34-41, 2006.

46. Campillo VE, Langonnet S, Pierrefeu A and Chaux-Bodard AG: Anatomic and histological study of the rabbit mandible as an experimental model for wound healing and surgical therapies. Lab Anim 48: 273-277, 2014. 
47. Namli H, Erdogan Ö, Gönlüsen G, Kahraman OE, Aydin HM, Karabag S and Tatli U: Vertical bone augmentation using bone marrow-derived stem cells: An in vivo study in the rabbit calvaria. Implant Dent 25: 54-62, 2016.

48. Polo CI, Sendyk WR, Correa L, Sendyk D, Deboni MCZ and Naclério-Homem MDG: Synergism between recombinant human bone morphogenetic protein 2/absorbable collagen sponge and bone substitutes favors vertical bone augmentation and the resorption rate of the biomaterials: Histomorphometric and 3D microcomputed tomography analysis. J Periodontol 91: 1295-1306, 2020.

49. Sudheesh Kumar PT, Hashimi S, Saifzadeh S, Ivanovski S and Vaquette C: Additively manufactured biphasic construct loaded with BMP-2 for vertical bone regeneration: A pilot study in rabbit. Mater Sci Eng C Mater Biol Appl 92: 554-564, 2018.

50. Lee JS, Lee JS, Kang MH, Jung UW, Choi SH and Cho KS: Proof-of-concept study of vertical augmentation using block-type allogenic bone grafts: A preclinical experimental study on rabbit calvaria. J Biomed Mater Res B Appl Biomater 106: 2700-2707, 2018.

51. Sheikh Z, Drager J, Zhang YL, Abdallah MN, Tamimi F and Barralet J: Controlling bone graft substitute microstructure to improve bone augmentation. Adv Healthc Mater 5: 1646-1655, 2016.

52. Sheikh Z, Chen G, Thévenin M, Young RN, Grynpas MD and Glogauer M: A novel anabolic conjugate (C3) in the matrix of dicalcium phosphate onlay block grafts for achieving vertical bone augmentation: An experimental study on rabbit calvaria. Int J Oral Maxillofac Implants 34: e51-e63, 2019.

53. Tamimi F, Torres J, Al-Abedalla K, Lopez-Cabarcos E, Alkhraisat MH, Bassett DC, Gbureck U and Barralet JE: Osseointegration of dental implants in 3D-printed synthetic onlay grafts customized according to bone metabolic activity in recipient site. Biomaterials 35: 5436-5445, 2014.

54. Draenert FG, Kammerer PW, Palarie V and Wagner W: Vertical bone augmentation with simultaneous dental implantation using crestal biomaterial rings: A rabbit animal study. Clin Implant Dent Relat Res 14 (Suppl 1): e169-e174, 2012.

55. Kim YJ, de Molon RS, Horiguti FR, Contador GP, Coelho MA, Mascarenhas VI, de Souza Faloni AP, Cirelli JA and Sendyk WR: Vertical bone augmentation using deproteinized bovine bone mineral, absorbable collagen sponge, and recombinant human bone morphogenetic protein-2: An in vivo study in rabbits. In J Oral Maxillofac Implants 33: 512-522, 2018.

56. Veis A, Dabarakis N, Koutrogiannis C, Barlas I, Petsa E and Romanos G: Evaluation of vertical bone regeneration using block and particulate forms of Bio-oss bone graft: A histologic study in the rabbit mandible. J Oral Implantol 41: e66-e72, 2015

57. Pripatnanont $P$, Balabid F, Pongpanich $S$ and Vongvatcharanon $S$ Effect of osteogenic periosteal distraction by a modified Hyrax device with and without platelet-rich fibrin on bone formation in a rabbit model: A pilot study. Int J Oral Maxillofac Surg 44: 656-663, 2015.

58. Thoma DS, Kruse A, Ghayor C, Jung RE and Weber FE: Bone augmentation using a synthetic hydroxyapatite/silica oxide-based and a xenogenic hydroxyapatite-based bone substitute materials with and without recombinant human bone morphogenetic protein-2. Clin Oral Implants Res 26: 592-598, 2015.

59. Lai CH, Zhou L, Wang ZL, Lu HB and Gao Y: Use of a collagen membrane loaded with recombinant human bone morphogenetic protein-2 with collagen-binding domain for vertical guided bone regeneration. J Periodontol 84: 950-957, 2013.

60. de Molon RS, Sakakura CE, Faeda RS, Sartori R, Palhares D, Margonar R and Marcantonio E Jr: Effect of the long-term administration of Cyclosporine A on bone healing around osseointegrated titanium implants: A histomorphometric study in the rabbit tibia. Microsc Res Tech 80: 1000-1008, 2017.

61. Cohen DJ, Cheng A, Kahn A, Aviram M, Whitehead AJ, Hyzy SL, Clohessy RM, Boyan BD and Schwartz Z: Novel osteogenic Ti-6Al-4V device for restoration of dental function in patients with large bone deficiencies: Design, development and implementation. Sci Rep 6: 20493, 2016.

62. Wancket LM: Animal models for evaluation of bone implants and devices: Comparative bone structure and common mode uses. Vet Pathol 52: 842-850, 2015.

63. Reitan K and Kvam E: Comparative behavior of human and animal tissue during experimental tooth movement. Angle Orthod 41: 1-14, 1971.

64. Kimmel DB and Jee WS: A quantitative histologic study of bone turnover in young adult beagles. Anat Rec 203: 31-45, 1982.
65. Carrel JP, Wiskott A, Scherrer S and Durual S: Large bone vertical augmentation using a three-dimensional printed TCP/HA bone graft: A pilot study in dog mandible. Clin Implant Dent Relat Res 18: 1183-1192, 2016.

66. Teng F, Wei L, Yu D, Deng L, Zheng Y, Lin H and Liu Y: Vertical bone augmentation with simultaneous implantation using deproteinized bovine bone block functionalized with a slow delivery of BMP-2. Clin Oral Implants Res 31: 215-228, 2020.

67. Schwarz F, Mihatovic I, Popal-Jensen I, Parvini P and Sader R: Influence of autoclavation on the efficacy of extracted tooth roots used for vertical alveolar ridge augmentation. J Clin Periodontol 46: 502-509, 2019.

68. Parvini P, Schwarz F, Hüfner MK, Rauch N, Nienkemper M and Becker K: Microstructural volumetric analysis of vertical alveolar ridge augmentation using autogenous tooth roots. Clin Implant Dent Relat Res 22: 647-653, 2020.

69. Parvini P, Schliephake C, Al-Maawi S, Schwarz K, Sader R, Ghanaati S and Schwarz F: Histomorphometrical assessment of vertical alveolar ridge augmentation using extracted tooth roots in the canine. Clin Oral Investig 24: 317-323, 2020.

70. Hsu YT, Al-Hezaimi K, Galindo-Moreno P, O'Valle F, Al-Rasheed A and Wang HL: Effects of recombinant human bone morphogenetic protein-2 on vertical bone augmentation in a canine model. J Periodontol 88: 896-905, 2017.

71. Xuan F, Lee CU, Son JS, Fang Y, Jeong SM and Choi BH: Vertical ridge augmentation using xenogenous bone blocks: A comparison between the flap and tunneling procedures. J Oral Maxillofac Surg 72: 1660-1670, 2014.

72. Esposito $M$, Trullenque-Eriksson A, Vila $C N$, Peñarrocha $M$, García A, Sánchez A, Muñoz-Guzón F and Martínez Martín JM: Vertical osseodistraction with a new intraosseous alveolar distractor prototype for dental implant rehabilitation: A pilot study in dogs. Int J Oral Maxillofac Implants 32: 838-848, 2017.

73. Terbish M, Yoo SH, Kim HJ, Yu HS, Hwang CJ, Baik HS and Cha JY: Accelerated bone formation in distracted alveolar bone after injection of recombinant human bone morphogenetic protein-2. J Periodontol 86: 1078-1086, 2015.

74. Kaner D, Zhao H, Terheyden H and Friedmann A: Improvement of microcirculation and wound healing in vertical ridge augmentation after pre-treatment with self-inflating soft tissue expanders-a randomized study in dogs. Clin Oral Implants Res 26: 720-724, 2015.

75. Kaner D, Zhao H, Arnold W, Terheyden H and Friedmann A: Pre-augmentation soft tissue expansion improves scaffold-based vertical bone regeneration-a randomized study in dogs. Clin Oral Implants Res 28: 640-647, 2017.

76. Sawada K, Nakahara K, Haga-Tsujimura M, Iizuka T, Fujioka-Kobayashi M, Igarashi K and Saulacic N: Comparison of three block bone substitutes for bone regeneration: Long-term observation in the beagle dog. Odontology 106: 398-407, 2018.

77. Bianchini MA, Pontual MA, Bez L, Benfatti CA, Boabaid F, Somerman MJ and Magini RS: The use of bovine screws to promote bone formation using a tibia model in dogs. Oral Surg Oral Med Oral Pathol Oral Radiol 116: e215-e220, 2013.

78. Pearce AI, Richards RG, Milz S, Schneider E and Pearce SG: Animal models for implant biomaterial research in bone: A review. Eur Cell Mater 13: 1-10, 2007.

79. Khojasteh A, Behnia H, Hosseini FS, Dehghan MM, Abbasnia P and Abbas FM: The effect of PCL-TCP scaffold loaded with mesenchymal stem cells on vertical bone augmentation in dog mandible: A preliminary report. J Biomed Mater Res B Appl Biomater 101: 848-854, 2013.

80. Mosekilde L, Weisbrode SE, Safron JA, Stills HF, Jankowsky ML, Ebert DC, Danielsen CC, Sogaard CH, Franks AF, Stevens ML, et al: Calcium-restricted ovariectomized Sinclair S-1 minipigs: An animal model of osteopenia and trabecular plate perforation. Bone 14: 379-382, 1993.

81. Harper RA, Pfeiffer FM and Choma TJ: The minipig as a potential model for pedicle screw fixation: Morphometry and mechanics. J Orthop Surg Res 14: 246, 2019.

82. Bonucci E and Ballanti P: Osteoporosis-bone remodeling and animal models. Toxicol Pathol 42: 957-969, 2014

83. Moest T, Koehler F, Prechtl C, Schmitt C, Watzek G and Schlegel KA: Bone formation in peri-implant defects grafted with microparticles: A pilot animal experimental study. J Clin Periodontol 41: 990-998, 2014.

84. Reichert JC, Saifzadeh S, Wullschleger ME, Epari DR, Schütz MA, Duda GN, Schell H, van Griensven M, Redl H and Hutmacher DW: The challenge of establishing preclinical models for segmental bone defect research. Biomaterials 30: 2149-2163, 2009. 
85. Schorn L, Sproll C, Ommerborn M, Naujoks C, Kübler NR and Depprich R: Vertical bone regeneration using rhBMP-2 and VEGF. Head Face Med 13: 11, 2017.

86. Moest T, Frabschka J, Kesting MR, Schmitt CM, Frohwitter G, Lutz R and Schlegel KA: Osseous ingrowth in allogeneic bone blocks applied for vertical bone augmentation: A preclinical randomised controlled study. Clin Oral Investig 24: 2867-2879, 2020.

87. Freilich M, Wen B, Shafer D, Schleier P, Dard M, Pendrys D, Ortiz D and Kuhn L: Implant-guided vertical bone growth in the mini-pig. Clin Oral Implants Res 23: 751-757, 2012.

88. Wen B, Shafer D, Schleier P, Pendrys D, Kuhn L and Freilich M: Implant-guided supracrestal alveolar bone growth using scaffolds, BMP-2, and novel scaffold-retaining device. Clin Oral Implants Res 28: 1411-1420, 2017.

89. Jinno $Y$, Jimbo R, Lindström M, Sawase T, Lilin T and Becktor JP: Vertical bone augmentation using ring technique with three different materials in the sheep mandible bone. Int J Oral Maxillofac Implants 33: 1057-1063, 2018.

90. Moussa M, Carrel JP, Scherrer S, Cattani-Lorente M, Wiskott A and Durual S: Medium-term function of a 3D printed TCP/HA structure as a new osteoconductive scaffold for vertical bone augmentation: A simulation by BMP-2 activation. Materials 8: 2174-2190, 2015

91. Eitel F, Klapp F, Jacobson W and Schweiberer L: Bone regeneration in animals and in man. A contribution to understanding the relative value of animal experiments to human pathophysiology. Arch Orthop Trauma Surg 99: 59-64, 1981.

92. Carrel JP, Wiskott A, Moussa M, Rieder P, Scherrer S and Durual S: A 3D printed TCP/HA structure as a new osteoconductive scaffold for vertical bone augmentation. Clin Oral Implants Res 27: 55-62, 2016.

93. Benlidayi ME, Tatli U, Salimov F, Tükel HC and Yüksel O Comparison of autogenous and allograft bone rings in surgically created vertical bone defects around implants in a sheep model. Clin Oral Implants Res 29: 1155-1162, 2018.

94. Çolpak HA, Gönen ZB, Özdamar S, Alkan A and Kütük N: Vertical ridge augmentation using guided bone regeneration procedure and dental pulp derived mesenchymal stem cells with simultaneous dental implant placement: A histologic study in a sheep model. J Stomatol Oral Maxillofac Surg 120: 216-223, 2019.
95. Hurtig MB, Buschmann MD, Fortier LA, Hoemann CD, Hunziker EB, Jurvelin JS, Mainil-Varlet P, McIlwraith CW, Sah RL and Whiteside RA: Preclinical studies for cartilage repair: Recommendations from the international cartilage repair society. Cartilage 2: 137-152, 2011.

96. Hoemann C, Kandel R, Roberts S, Saris DB, Creemers L, Mainil-Varlet P, Méthot S, Hollander AP and Buschmann MD: International cartilage repair society (ICRS) recommended guidelines for histological endpoints for cartilage repair studies in animal models and clinical trials. Cartilage 2: 153-172, 2011

97. Polo CI, Lima JL, De Lucca L, Piacezzi CB Naclério-Homem Mda G, Arana-Chavez VE and Sendyk WR: Effect of recombinant human bone morphogenetic protein 2 associated with a variety of bone substitutes on vertical guided bone regeneration in rabbit calvarium. J Periodontol 84: 360-370, 2013.

98. Greenstein G, Greenstein B, Cavallaro J and Tarnow D: The role of bone decortication in enhancing the results of guided bone regeneration: A literature review. J Periodontol 80: 175-189, 2009.

99. Kim DH, Cha JK, Song YW, Woo KM and Jung UW: Bone augmentation using small molecules with biodegradable calcium sulfate particles in a vertical onlay graft model in the rabbit calvarium. J Biomed Mater Res B Appl Biomater 108: 1343-1350, 2020.

100. Kim JW, Jung IH, Lee KI, Jung UW, Kim CS, Choi SH, Cho KS and Yun JH: Volumetric bone regenerative efficacy of biphasic calcium phosphate-collagen composite block loaded with rhBMP-2 in vertical bone augmentation model of a rabbit calvarium. J Biomed Mater Res A 100: 3304-3313, 2012.

101. Zere E, Einy S, Asbi T, Aizenbud Y, Gutmacher Z, Katzhandler E and Aizenbud D: Orthodontic extraction space closure with and without socket preservation: A comparative case analysis. Quintessence Int 50: 306-314, 2019. International (CC BY-NC-ND 4.0) License. 